\title{
ON AN EXAMPLE CONCERNING THE SECOND RIGIDITY THEOREM
}

\author{
OLGUR CELIKBAS, HIROKI MATSUI, AND ARASH SADEGHI
}

\begin{abstract}
In this paper we revisit an example of Celikbas and Takahashi concerning the reflexivity of tensor products of modules. We study Tor-rigidity and the Hochster-Huneke graph with vertices consisting of minimal prime ideals, and determine a condition with which the aforementioned example cannot occur. Our result, in particular, corroborates the Second Rigidity Theorem of Huneke and Wiegand.
\end{abstract}

\section{INTRODUCTION}

Throughout $R$ denotes a commutative Noetherian local ring with unique maximal ideal $\mathfrak{m}$, and all $R$-modules are assumed to be finitely generated. For unexplained notations and terminology, such as the definitions of homological dimensions, we refer the reader to [4, 6, 20].

In this paper we are concerned with the following result of Huneke and Wiegand, which is known as the Second Rigidity Theorem; see [16, 2.1].

Theorem 1.1. (Huneke and Wiegand [16]) Let $R$ be a hypersurface ring, and let $M$ and $N$ be $R$-modules such that $M$ has rank, i.e., there is a nonnegative integer $r$ such that $M_{\mathfrak{p}}$ is free of rank $r$ for each associated prime ideal $\mathfrak{p}$ of $R\left(\right.$ e.g., $\left.\operatorname{pd}_{R}(M)<\infty\right)$. If $M \otimes_{R} N$ is reflexive, or in this context equivalently, is a second syzygy module, then $N$ is reflexive.

Another conclusion of Theorem 1.1, which is worth noting, is the vanishing of $\operatorname{Tor}_{i}^{R}(M, N)$ for each $i \geq 1$. For quite some time it has been an open problem whether the module $M$ in Theorem 1.1 must also be reflexive; see [18]. Recently Celikbas and Takahashi [10] has given an example disproving this query: there is a reduced hypersurface ring $R$, and modules $M$ and $N$ over $R$ such that both $M \otimes_{R} N$ and $N$ are reflexive, $\operatorname{pd}_{R}(M)<\infty$, but $M$ is not reflexive. Moreover, it can be easily checked that there exists a prime ideal $\mathfrak{q}$ of $R$ of height one such that the module $N$ in the example satisfies $\operatorname{pd}_{R_{\mathfrak{q}}}\left(N_{\mathfrak{q}}\right)=\infty$; see Example 4.5 for details. The main aim of this paper is to show that such an example cannot occur in case $\operatorname{pd}_{R_{\mathfrak{p}}}\left(N_{\mathfrak{p}}\right)<\infty$ for each prime ideal $\mathfrak{p}$ of $R$ of height at most one. More precisely, we prove:

Theorem 1.2. Assume $R$ is a hypersurface ring (quotient of an unramified regular local ring), and $M$ and $N$ are nonzero R-modules. Assume further:

(i) $\operatorname{pd}(M)<\infty$.

(ii) $\operatorname{pd}_{R_{\mathfrak{p}}}\left(N_{\mathfrak{p}}\right)<\infty$ for each prime ideal $\mathfrak{p}$ of $R$ of height at most one.

If $M \otimes_{R} N$ is reflexive, then both $M$ and $N$ are reflexive.

2010 Mathematics Subject Classification. Primary 13D07; Secondary 13H10, 13D05, 13C12.

Key words and phrases. Reflexivity of tensor products of modules, Serre's condition, syzygy, vanishing of Tor.

Sadeghi's research was supported by a grant from the Institute for Research in Fundamental Sciences (IPM). Matsui's research was supported by JSPS Grant-in-Aid for Young Scientists 19J00158. 
We give a proof of Theorem 1.2 in section 4, but in fact our main argument is more general: we consider tensor products $M \otimes_{R} N$ which are $n$-th syzygy modules for $n \geq 2$ and modules $N$ of finite complete intersection dimension over rings that are not necessarily hypersurfaces; see Theorem 3.1. A key ingredient of our proof is the fact that, when $R$ satisfies Serre's condition $\left(S_{2}\right)$, the Hochster-Huneke graph [15] is connected; see Theorem 4.3 ,

\section{Preliminaries}

2.1. An $R$-module $M$ is said to be Tor-rigid provided that the following condition holds: if $N$ is an $R$-module with $\operatorname{Tor}_{1}^{R}(M, N)=0$, then $\operatorname{Tor}_{2}^{R}(M, N)=0$. Examples of Tor-rigid modules are abundant in the literature. For example, each syzgy of the $R$-module $M$ is Tor-rigid if:

(i) $R$ is a hypersurface that is quotient of an unramified regular local ring, and $M$ has either finite length or finite projective dimension; see [16, 2.4] and [19, Theorem 3].

(ii) $R$ has positive depth and $M=\mathfrak{m}^{r}$ for some integer $r \geq 1$; see [11, 2.5].

2.2. Let $M$ be an $R$-module with a projective presentation $P_{1} \stackrel{f}{\rightarrow} P_{0} \rightarrow M \rightarrow 0$. The transpose $\operatorname{Tr} M$ of $M$ is the cokernel of $f^{*}=\operatorname{Hom}_{R}(f, R)$, and hence is given by the exact sequence: $0 \rightarrow M^{*} \rightarrow P_{0}^{*} \rightarrow P_{1}^{*} \rightarrow \operatorname{Tr} M \rightarrow 0$. Note $\operatorname{Tr} M$ is well-defined up to projective summands.

Given an integer $n \geq 0$, it follows from [2, 2.8] that there is an exact sequence of functors:

$0 \rightarrow \operatorname{Ext}_{R}^{1}\left(\operatorname{Tr} \Omega^{n} M,-\right) \rightarrow \operatorname{Tor}_{n}^{R}(M,-) \rightarrow \operatorname{Hom}_{R}\left(\operatorname{Ext}_{R}^{n}(M, R),-\right) \rightarrow \operatorname{Ext}_{R}^{2}\left(\operatorname{Tr} \Omega^{n} M,-\right)$.

Recall that an $R$-module $N$ is said to be torsionless if the natural map $N \rightarrow N^{* *}$ is injective, i.e., $\operatorname{Ext}_{R}^{1}(\operatorname{Tr} N, R)=0$; see 2.2.

2.3. Let $N$ be a torsionless $R$-module and let $\left\{f_{1}, f_{2}, \ldots, f_{s}\right\}$ be a minimal generating set of the module $N^{*}=\operatorname{Hom}(N, R)$. Let $\delta: R^{\oplus s} \rightarrow N^{*}$ be defined by $\delta\left(e_{i}\right)=f_{i}$ for $i=1,2, \ldots, s$, where $\left\{e_{1}, e_{2}, \ldots, e_{s}\right\}$ is the standard basis for $R^{\oplus s}$. Then, composing the natural injective map $N \hookrightarrow N^{* *}$ with $\delta^{*}$, we obtain the short exact sequence:

$$
0 \rightarrow N \stackrel{u}{\rightarrow} R^{\oplus s} \rightarrow N_{1} \rightarrow 0,
$$

where $u(x)=\left(f_{1}(x), f_{2}(x), \ldots, f_{s}(x)\right)$ for all $x \in N$; see 2.2. Any module $N_{1}$ obtained in this way is called a pushforward (or left projective approximation) of $M$; see [3, 13]. Note that such a construction is unique, up to a non-canonical isomorphism; see, for example, [13, page 62]. Also it follows $\operatorname{Ext}_{R}^{1}\left(N_{1}, R\right)=0$ so that $\Omega \operatorname{Tr} N \cong \operatorname{Tr} N_{1}$ (up to free summands); see [2, 3.9].

2.4. Let $M$ be an $R$-module and let $n \geq 0$ be an integer. Then $M$ is said to satisfy $\left(\widetilde{S_{n}}\right)$ provided $\operatorname{depth}_{R_{\mathfrak{q}}}\left(M_{\mathfrak{q}}\right) \geq \min \left\{n, \operatorname{depth}\left(R_{\mathfrak{q}}\right)\right\}$ for each $\mathfrak{q} \in \operatorname{Supp}(M)$ (note $\left.\operatorname{depth}(0)=\infty\right)$ If $R$ is CohenMacaulay, then $M$ satisfies $\left(\widetilde{S_{n}}\right)$ if and only if $M$ satisfies Serre's condition $\left(S_{n}\right)$; see [13].

2.5. Given an integer $s \geq 0$, we set $Y^{s}(R)=\left\{\mathfrak{p} \in \operatorname{Spec} R \mid \operatorname{depth}\left(R_{\mathfrak{p}}\right) \leq s\right\}$. In particular, $\mathrm{Y}^{0}(R)$ denotes the set of all associated prime ideals of $R$.

2.6. ([12, 2.4] and [13,3.8]) Let $M$ be an $R$-module and let $n \geq 1$ be an integer. Assume that $\mathrm{G}-\operatorname{dim}_{R_{\mathfrak{p}}}\left(M_{\mathfrak{p}}\right)<\infty$ for each $\mathfrak{p} \in \mathrm{Y}^{n-1}(R)$. Then the following conditions are equivalent:

(i) $M$ satisfies $\left(\widetilde{S_{n}}\right)$.

(ii) $M$ is $n$-torsion-free, i.e., $\operatorname{Ext}_{R}^{i}(\operatorname{Tr} M, R)=0$ for each $i=1, \ldots, n$.

(iii) $M$ is an $n$-th syzygy module, i.e., $M \cong \Omega^{n}(N)$ for some $R$-module $N$. 
2.7. Let $M$ and $N$ be $R$-modules with $\mathrm{Cl}-\operatorname{dim}(M)<\infty$ or $\mathrm{Cl}-\operatorname{dim}(N)<\infty$. If $\operatorname{Tor}_{i}^{R}(M, N)=0$ for each $i \geq 1$, then $\operatorname{depth}(M)+\operatorname{depth}(N)=\operatorname{depth}(R)+\operatorname{depth}\left(M \otimes_{R} N\right)$, i.e., the depth formula holds; see [1, 2.5].

2.8. Let $M$ and $N$ be $R$-modules such that $\mathrm{Cl}-\operatorname{dim}(N)=0$. Then $\operatorname{Ext}_{R}^{i}(\operatorname{Tr} N, M)=0$ for all $i \geq 1$ if and only if $\operatorname{Tor}_{i}^{R}(M, N)=0$ for all $i \geq 1$; see [9, 3.2].

\section{MAIN THEOREM}

In this section we will prove the following theorem which is our main result:

Theorem 3.1. Let $M$ and $N$ be nonzero $R$-modules, and let $n \geq 1$ be an integer. Assume:

(i) $M$ is Tor-rigid.

(ii) $\mathrm{Cl}-\operatorname{dim}(N)<\infty$.

(iii) $M \otimes_{R} N$ satisfies $\left(\widetilde{S_{n}}\right)$.

(iv) $\operatorname{Tor}_{i}^{R}(M, N)$ is torsion for all $i \gg 0$.

Then $\operatorname{Tor}_{i}^{R}(M, N)=0$ for all $i \geq 1$, and $N$ satisfies $\left(\widetilde{S_{n}}\right)$.

To prove Theorem 3.1, we will establish several lemmas.

Lemma 3.2. Let $0 \rightarrow N \stackrel{\mu}{\longrightarrow} F \rightarrow N_{1} \rightarrow 0$ be a short exact sequence of $R$-modules, where $F$ is free and $\operatorname{Ext}_{R}^{1}\left(N_{1}, R\right)=0$. Then it follows that $\operatorname{Ext}_{R}^{1}(\operatorname{Tr} N, M) \cong \operatorname{Tor}_{1}^{R}\left(N_{1}, M\right)$.

Proof. We consider the following commutative diagram, where the horizontal maps are the natural ones and $\operatorname{Hom}\left(\mu^{*}, M\right)$ is injective:

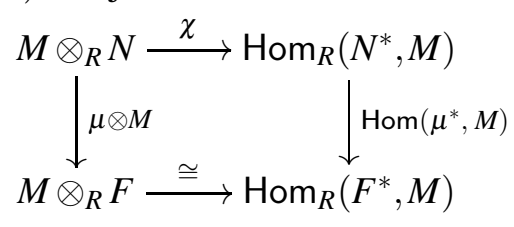

Note that $\operatorname{Ext}_{R}^{1}(\operatorname{Tr} N, M)=\operatorname{ker}(\chi)$; see 2.2. Hence it follows from the above diagram that $\operatorname{Ext}_{R}^{1}(\operatorname{Tr} N, M)=\operatorname{ker}(\chi) \cong \operatorname{ker}(\mu \otimes M)=\operatorname{Tor}_{1}^{R}\left(N_{1}, M\right)$, as required.

Lemma 3.3. Let $M$ and $N$ be $R$-modules with $\mathrm{Cl}-\operatorname{dim}_{R}(N)<\infty$. If $\operatorname{Tor}_{i}^{R}(M, N)$ is torsion for all $i \gg 0$, then $\operatorname{Tor}_{i}^{R}(M, N)$ and $\operatorname{Ext}_{R}^{i}(\operatorname{Tr} N, M)$ are torsion for each $i \geq 1$; cf., [7, A.2.].

Proof. Let $\mathfrak{p} \in \mathrm{Y}^{0}(R)$. Then, since $\operatorname{Tor}_{i}^{R_{\mathfrak{p}}}\left(M_{\mathfrak{p}}, N_{\mathfrak{p}}\right)=0$ for all $i \gg 0$ and $\mathrm{Cl}$-dim $\operatorname{dim}_{R_{\mathfrak{p}}}\left(N_{\mathfrak{p}}\right)=0$, we conclude that $\operatorname{Tor}_{i}^{R_{\mathfrak{p}}}\left(M_{\mathfrak{p}}, N_{\mathfrak{p}}\right)=0$ for all $i \geq 1$ and also $\operatorname{Ext}_{R_{\mathfrak{p}}}^{i}\left(\operatorname{Tr}_{R_{\mathfrak{p}}} N_{\mathfrak{p}}, M_{\mathfrak{p}}\right)=0$ for all $i \geq 1$; see 2.8] and [5, 4.9].

Lemma 3.4. Let $M$ and $N$ be $R$-modules such that $M \neq 0$ and $M$ is Tor-rigid. If $n \geq 1$ is an integer and $\operatorname{Ext}_{R}^{n}(N, M)=0$, then $\operatorname{Ext}_{R}^{n}(N, R)=0$.

Proof. It follows from [2, 2.8(b)] that there is an exact sequence:

$$
\operatorname{Tor}_{2}^{R}\left(\operatorname{Tr} \Omega^{n} N, M\right) \rightarrow \operatorname{Ext}_{R}^{n}(N, R) \otimes_{R} M \rightarrow \operatorname{Ext}_{R}^{n}(N, M) \rightarrow \operatorname{Tor}_{1}^{R}\left(\operatorname{Tr} \Omega^{n} N, M\right) \rightarrow 0 .
$$

As $\operatorname{Ext}_{R}^{n}(N, M)=0$ and $M$ is Tor-rigid, we have that $\operatorname{Tor}_{2}^{R}\left(\operatorname{Tr} \Omega^{n} N, M\right)=0$. Thus we conclude $\operatorname{Ext}_{R}^{n}(N, R) \otimes_{R} M \cong \operatorname{Ext}_{R}^{n}(N, M)=0$. This gives, since $M \neq 0$, that $\operatorname{Ext}_{R}^{n}(N, R)=0$.

We are now ready to give a proof for our main result: 
Proof of Theorem 3.1 Note, to show $N$ satisfies $\left(\widetilde{S_{n}}\right)$, in view of 2.6 and Lemma 3.4, it suffices to prove $\operatorname{Ext}_{R}^{i}(\operatorname{Tr} N, M)=0$ for each $i=1, \ldots, n$. The vanishing of $\operatorname{Ext}_{R}^{i}(\operatorname{Tr} N, M)$, as well as that $\operatorname{Tor}_{i}^{R}(M, N)$, is clear if $\operatorname{depth}(R)=0$; see Lemma 3.3. So we assume $\operatorname{depth}(R) \geq 1$.

It follows from 2.2 that there is an injection: $\operatorname{Ext}_{R}^{1}(\operatorname{Tr} N, M) \hookrightarrow M \otimes_{R} N$. It is easy to see, since $M \otimes_{R} N$ satisfies $\left(\widetilde{S_{1}}\right)$, that $M \otimes_{R} N$ is torsion-free. On the other hand, $\operatorname{Ext}_{R}^{1}(\operatorname{Tr} N, M)$ is torsion; see Lemma 3.3. This establishes the theorem for the case where $n=1$, and also yield the vanishing of $\operatorname{Tor}_{i}^{R}(M, N)$ as we observe next: it follows from Lemma3.4 that $\operatorname{Ext}_{R}^{1}(\operatorname{Tr} N, R)=0$, and hence we can consider the pushforward $N_{1}$ of $N$; see 2.6 and 2.3. Now Lemma 3.2 shows $\operatorname{Ext}_{R}^{1}(\operatorname{Tr} N, M)=0=\operatorname{Tor}_{1}^{R}\left(N_{1}, M\right)$. As $M$ is Tor-rigid, we have $\operatorname{Tor}_{i}^{R}(M, N)=0$ for each $i \geq 1$.

Next we assume $n \geq 2$, and proceed by induction on $n$ to show that $N$ satisfies $\left(\widetilde{S_{n}}\right)$. Suppose there is an integer $t$ such that $t<n$ and $\operatorname{Ext}_{R}^{i}(\operatorname{Tr} N, M)=0$ for each $i=1, \ldots, t$. Our aim is to prove the vanishing of $\operatorname{Ext}_{R}^{t+1}(\operatorname{Tr} N, M)$.

It follows from Lemma 3.4 that $\operatorname{Ext}_{R}^{i}(\operatorname{Tr} N, R)=0$ for each $i=1, \ldots, t$, i.e., $N$ satisfies $\left(\widetilde{S_{t}}\right)$. Therefore we can consider the pushforward sequences

$$
0 \rightarrow N_{i-1} \rightarrow F_{i} \rightarrow N_{i} \rightarrow 0
$$

where $N_{0}=N, F_{i}$ is free and $\operatorname{Ext}_{R}^{1}\left(N_{i}, R\right)=0$ for each $i=1, \ldots, t$; see 2.3.

Note that, for each $i=1, \ldots, t$, we have:

$$
\operatorname{Tor}_{1}^{R}\left(M, N_{i}\right) \cong \operatorname{Ext}_{R}^{1}\left(\operatorname{Tr} N_{i-1}, M\right) \cong \operatorname{Ext}_{R}^{i}(\operatorname{Tr} N, M)=0 .
$$

Here, the first isomorphism in $3.1,2)$ is due to Lemma 3.2, while the second isomorphism follows since $\Omega^{i-1} \operatorname{Tr} N \cong \operatorname{Tr} N_{i-1}$ for $i=1, \ldots t$; see 2.3 .

Now, in view of (3.12), tensoring the short exact sequences in $3.1,1)$ with $M$, we obtain the following short exact sequences for each $i=1, \ldots, t$ :

$$
0 \rightarrow M \otimes_{R} N_{i-1} \rightarrow M \otimes_{R} F_{i} \rightarrow M \otimes_{R} N_{i} \rightarrow 0 .
$$

Recall our aim is to show that $\operatorname{Ext}_{R}^{t+1}(\operatorname{Tr} N, M)=0$, and since $\Omega^{t} \operatorname{Tr} N \cong \operatorname{Tr} N_{t}$ (up to free summands), we have $\operatorname{Ext}_{R}^{t+1}(\operatorname{Tr} N, M) \cong \operatorname{Ext}_{R}^{1}\left(\operatorname{Tr} N_{t}, M\right)$; see 2.3. So 2.2 yields an injection as:

$$
\mathrm{Ext}_{R}^{t+1}(\operatorname{Tr} N, M) \hookrightarrow M \otimes_{R} N_{t} .
$$

Next we assume $\operatorname{Ext}_{R}^{t+1}(\operatorname{Tr} N, M) \neq 0$, pick $\mathfrak{q} \in \operatorname{Ass}\left(\operatorname{Ext}_{R}^{t+1}(\operatorname{Tr} N, M)\right)$, and seek a contradiction.

Suppose $\mathfrak{q} \in \mathrm{Y}^{t}(R)$. Then, since $N$ satisfies $\left(\widetilde{S}_{t}\right)$, we have $\operatorname{depth}_{R_{\mathfrak{q}}}\left(N_{\mathfrak{q}}\right) \geq \operatorname{depth}\left(R_{\mathfrak{q}}\right)$. This shows $C$ - $\operatorname{dim}_{R_{\mathfrak{q}}}\left(N_{\mathfrak{q}}\right)=\operatorname{depth}\left(R_{\mathfrak{q}}\right)-\operatorname{depth}_{R_{\mathfrak{q}}}\left(N_{\mathfrak{q}}\right)=0$. Therefore, since $\operatorname{Tor}_{i}^{R}(M, N)=0$ for each $i \geq 1$, we deduce from 2.8 that $\operatorname{Ext}_{R}^{i}(\operatorname{Tr} N, M)_{\mathfrak{q}}=0$ for each $i \geq 1$. In particular $\mathfrak{q} \notin \mathrm{Y}^{t}(R)$, i.e., $\operatorname{depth}\left(R_{\mathfrak{q}}\right) \geq t+1$, because of the fact that $\operatorname{Ext}_{R}^{t+1}(\operatorname{Tr} N, M)_{\mathfrak{q}} \neq 0$.

Notice $\mathfrak{q} \in \operatorname{Supp}(M) \cap \operatorname{Supp}(N)$. Hence it follows from 2.7 that

$$
\text { 3.1.5) } \quad \operatorname{depth}_{R_{\mathfrak{q}}}\left(M_{\mathfrak{q}}\right)=\left(\operatorname{depth}\left(R_{\mathfrak{q}}\right)-\operatorname{depth}_{R_{\mathfrak{q}}}\left(N_{\mathfrak{q}}\right)\right)+\operatorname{depth}_{R_{\mathfrak{q}}}\left(M_{\mathfrak{q}} \otimes_{R_{\mathfrak{q}}} N_{\mathfrak{q}}\right) \geq t+1 \text {. }
$$

The inequality in 3.15) are due to the following facts: $t+1 \leq n$ so that $M \otimes_{R} N$ satisfies $\left(\widetilde{S}_{t+1}\right)$, $\operatorname{depth}\left(R_{\mathfrak{q}}\right) \geq t+1$, and Cl-dim $R_{R_{\mathfrak{q}}}\left(N_{\mathfrak{q}}\right)=\operatorname{depth}\left(R_{\mathfrak{q}}\right)-\operatorname{depth}_{R_{\mathfrak{q}}}\left(N_{\mathfrak{q}}\right) \geq 0$.

Recall that $\operatorname{Ext}_{R}^{t+1}(\operatorname{Tr} N, M)_{\mathfrak{q}}$ is a nonzero module of depth zero. Hence, we see, by revisiting (3.1.4), that $\operatorname{depth}_{R_{\mathfrak{q}}}\left(M_{\mathfrak{q}} \otimes_{R_{\mathfrak{q}}}\left(N_{t}\right)_{\mathfrak{q}}\right)=0$. However, by localizing (3.1,3) at $\mathfrak{q}$ and using depth lemma, along with (3.15), we have depth ${ }_{R_{\mathfrak{q}}}\left(M_{\mathfrak{q}} \otimes_{R_{\mathfrak{q}}} N_{\mathfrak{q}}\right)=t$; this is a contradiction since $M \otimes_{R}$ $N$ satisfies $\left(\widetilde{S}_{t+1}\right)$ and so depth $R_{\mathfrak{q}}\left(M_{\mathfrak{q}} \otimes_{R_{\mathfrak{q}}} N_{\mathfrak{q}}\right) \geq t+1$. Consequently, $\operatorname{Ext}_{R}^{t+1}(\operatorname{Tr} N, M)$ must vanish, and this completes the proof of the theorem. 


\section{PROOF OF THEOREM 1.2 AND FURTHER REMARKS}

Definition 4.1. ([15]) The Hochster-Huneke graph $G(R)$ is defined as follows:

- The set of vertices equals $\operatorname{Min}(R)$, i.e., vertices are the minimal prime ideals of $R$.

- There is an edge between two vertices $\mathfrak{p}$ and $\mathfrak{q}$ of $G(R) \Longleftrightarrow$ height $(\mathfrak{p}+\mathfrak{q}) \leq 1$.

Remark 4.2. ([15]) The following hold for the graph $G(R)$ :

(i) Given two vertices $\mathfrak{p}_{1}$ and $\mathfrak{p}_{2}$ of $G(R)$, there is an edge between $\mathfrak{p}_{1}$ and $\mathfrak{p}_{2}$ if and only if $\mathfrak{p}_{1}+\mathfrak{p}_{2}$ is contained in some height-one prime ideal $\mathfrak{q}$.

(ii) $G(R)$ is connected if and only if given two vertices $\mathfrak{p}$ and $\mathfrak{p}^{\prime}$ of $G(R)$, there are minimal prime ideals $\left\{\mathfrak{p}_{0}, \mathfrak{p}_{1}, \ldots, \mathfrak{p}_{r}\right\}$ of $R$, and height-one prime ideals $\left\{\mathfrak{q}_{1}, \mathfrak{q}_{2}, \ldots, \mathfrak{q}_{r}\right\}$ of $R$, where $\mathfrak{p}=\mathfrak{p}_{0}, \mathfrak{p}^{\prime}=\mathfrak{p}_{r}$ and $\mathfrak{p}_{i}, \mathfrak{p}_{i+1} \subseteq \mathfrak{q}_{i+1}$ for each $i=0,1, \ldots, r-1$.

The first part of the next proposition is proved in [15, 3.6] for complete local rings. Here, for the convenience of the reader, we go over its proof since we do not assume $R$ is complete.

Proposition 4.3. Assume $R$ satisfies $\left(S_{2}\right)$, e.g., $R$ is Cohen-Macaulay. Then the following hold:

(i) $G(R)$ is connected.

(ii) If $N$ is an $R$-module such that $N_{\mathfrak{p}}$ is free for each $\mathfrak{p} \in Y^{1}(R)$, then $N$ has rank.

Proof. (i) We assume $G(R)$ is not connected, and seek a contradiction.

Notice, since $G(R)$ is disconnected, there is a nontrivial partition of the set of all minimal prime ideals of $R$ as $\operatorname{Min}(R)=\left\{\mathfrak{p}_{1}, \cdots, \mathfrak{p}_{r}\right\} \sqcup\left\{\mathfrak{q}_{1}, \cdots, \mathfrak{q}_{s}\right\}$, where height $\left(\mathfrak{p}_{i}+\mathfrak{q}_{j}\right) \geq 2$ for each $i$ and $j$. Letting $I=\bigcap_{i=1}^{r} \mathfrak{p}_{i}$ and $J=\bigcap_{j=1}^{s} \mathfrak{q}_{j}$, we get two non-nilpotent ideals $I$ and $J$ such that $I J$ is nilpotent. Moreover it follows that height $(I+J) \geq 2$ since

$V(I+J)=V(I) \cap V(J)=\left[\bigcup_{i=1}^{r} V\left(\mathfrak{p}_{i}\right)\right] \bigcap\left[\bigcup_{j=1}^{s} V\left(\mathfrak{q}_{j}\right)\right]=\bigcup_{i, j} V\left(\mathfrak{p}_{i}+\mathfrak{q}_{j}\right)$ and height $\left(\mathfrak{p}_{i}+\mathfrak{q}_{j}\right) \geq 2$.

By replacing the ideals $I$ and $J$ with their appropriate powers, we may assume $I J=0$.

Since $R$ satisfies $\left(S_{2}\right)$ and height $(I+J) \geq 2$, there is an $R$-regular sequence $\left\{u+v, u^{\prime}+v^{\prime}\right\}$ in $I+J$, where $u, u^{\prime} \in I$ and $v, v^{\prime} \in J$. In view of the fact $v^{\prime}(u+v)-v\left(u^{\prime}+v^{\prime}\right)=v^{\prime} u-v u^{\prime} \in I J=0$, we conclude that there is an element $a \in R$ such that $v=a(u+v)$. Similarly, we deduce that $u=b(u+v)$ for some $b \in R$. Therefore we have $u+v=(a+b)(u+v)$, and hence $a+b$ is unit in $R$. This implies that either $a$ or $b$ is unit in $R$. We assume, without loss of generality, that $a$ is unit. Then $u$ is $R$-regular, and the equality $u J=0$ shows that $J=0$, which is a contradiction. Consequently, $G(R)$ is not connected.

(ii) Note, as $R$ satisfies $\left(S_{2}\right)$, each associated prime of $R$ is minimal, and $\mathfrak{p} \in Y^{1}(R)$ if and only if height $(\mathfrak{p}) \leq 1$. Moreover, by part (i), we know $G(R)$ is connected.

Let $\mathfrak{p}$ and $\mathfrak{p}^{\prime}$ be two minimal prime ideals of $R$. Then we know there are minimal prime ideals $\left\{\mathfrak{p}_{0}, \mathfrak{p}_{1}, \ldots, \mathfrak{p}_{r}\right\}$ of $R$, and height-one prime ideals $\left\{\mathfrak{q}_{1}, \mathfrak{q}_{2}, \ldots, \mathfrak{q}_{r}\right\}$ of $R$, where $\mathfrak{p}=\mathfrak{p}_{0}$, $\mathfrak{p}^{\prime}=\mathfrak{p}_{r}$ and $\mathfrak{p}_{i}, \mathfrak{p}_{i+1} \subseteq \mathfrak{q}_{i+1}$ for each $i=0,1, \ldots, r-1$.

By assumption, for each $i=0,1, \ldots, r-1$, we know that the modules $M_{\mathfrak{p}_{i}}, M_{\mathfrak{p}_{i+1}}$ and $M_{\mathfrak{q}_{i+1}}$ are free. Moreover, as $\left(M_{\mathfrak{q}_{i+1}}\right)_{\mathfrak{p}_{i} R_{\mathfrak{q}_{i+1}}} \cong M_{\mathfrak{p}_{i}}$, for each $i=0,1, \ldots, r-1$, we deduce:

$$
\operatorname{rank}_{R_{\mathfrak{p}_{i}}}\left(M_{\mathfrak{p}_{i}}\right)=\operatorname{rank}_{R_{\mathfrak{q}_{i+1}}}\left(M_{\mathfrak{q}_{i+1}}\right)=\operatorname{rank}_{R_{\mathfrak{p}_{i+1}}}\left(M_{\mathfrak{p}_{i+1}}\right) .
$$

This shows that $\operatorname{rank}_{R_{\mathfrak{p}}}\left(M_{\mathfrak{p}}\right)=\operatorname{rank}_{R_{\mathfrak{p}^{\prime}}}\left(M_{\mathfrak{p}^{\prime}}\right)$, as required. 
We can strengthen the conclusion of Theorem 3.1, and show that both modules in question satisfy $\left(\widetilde{S_{n}}\right)$ in case local freeness hypothesis on $\mathrm{Y}^{1}(R)$ is included in our assumptions.

Corollary 4.4. Assume $R$ satisfies $\left(S_{2}\right), n$ is a positive integer, and $M$ and $N$ are nonzero $R$-modules. Assume further:

(i) $M$ is Tor-rigid.

(ii) $\mathrm{Cl}-\operatorname{dim}(N)<\infty$.

(iii) $M \otimes_{R} N$ satisfies $\left(\widetilde{S_{n}}\right)$.

(iv) $\operatorname{pd}_{R_{\mathfrak{p}}}\left(N_{\mathfrak{p}}\right)<\infty$ for each $\mathfrak{p} \in \mathrm{Y}^{1}(R)$.

Then $\operatorname{Tor}_{i}^{R}(M, N)=0$ for all $i \geq 1$, and both $M$ and $N$ satisfy $\left(\widetilde{S_{n}}\right)$.

Proof. It follows from Theorem 3.1 that $\operatorname{Tor}_{i}^{R}(M, N)=0$ for all $i \geq 1$, and $N$ satisfies $\left(\widetilde{S_{n}}\right)$. Note that, both $M \otimes_{R} N$ and $N$ satisfy $\left(S_{1}\right)$. Hence $N_{\mathfrak{p}}$ is free for each $\mathfrak{p} \in \mathrm{Y}^{1}(R)$. In particular, $N$ has rank due to Proposition 4.3(ii). Therefore, since $M \otimes_{R} N$ is torsion-free, we conclude that $\operatorname{Supp}(N)=\operatorname{Spec}(R)$. Now the depth formula shows $M$ satisfies $\left(\widetilde{S_{n}}\right)$; see 2.7 and [8, 1.3].

Now we can prove Theorem 1.2, the result advertised in the introduction:

Proof of Theorem 1.2. The result is an immediate consequence of Corollary 4.4 since $M$ is Tor-rigid by a result of Lichtenbaum; see 2.1(i).

Next we recall an example given in [10] concerning the Second Rigidity Theorem; see Theorem 1.1. The presentation we provide for $M \otimes_{R} N$ in Example 4.5 has not been given in [10] and appears to be new; here we compute it by using [14, 21].

Example 4.5. ([10]) Let $R=\mathbb{C}[\mid x, y, z, w]] /(x y), M=\operatorname{Tr}(R / \mathfrak{p})$, where $\mathfrak{p}=(y, z, w) \in \operatorname{Spec}(R)$, and let $N=R /(x)$. Then $M$ is not reflexive, but since $\operatorname{pd}(M)<\infty$, we have that $N$ is reflexive by Theorem 1.1. Moreover, $M \otimes_{R} N$ is reflexive since it is the second syzygy of the cokernel of the rightmost matrix in the following exact sequence:

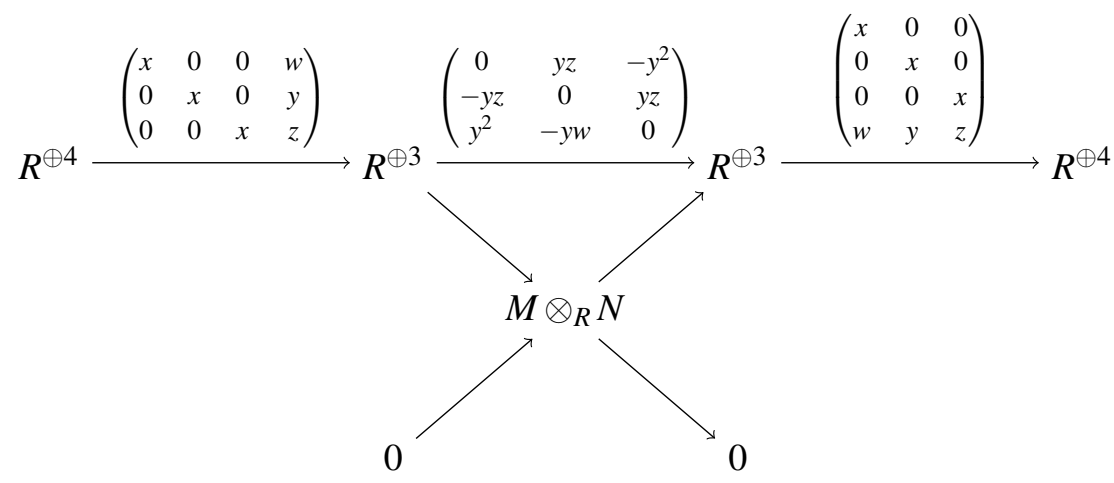

Next we point out that the conclusion of Theorem 1.2 is sharp:

Remark 4.6. In Example 4.5, it follows, as $\operatorname{pd}(M)<\infty$, that $\operatorname{Tor}_{i}^{R}(M, N)_{\mathfrak{p}}=0$ for all $i \gg 0$ and for all $\mathfrak{p} \in \operatorname{Spec}(R)$, but $M$ is not reflexive. In other words, the torsion hypothesis (iv) of Theorem 3.1 is not enough to obtain the conclusion of Theorem 1.2 , in general. 
We can easily see that there is a height-one prime ideal $\mathfrak{q}$ of $R$ in Example 4.5 such that $\operatorname{pd}_{R_{\mathfrak{q}}}\left(N_{\mathfrak{q}}\right)=\infty$. For that note the minimal free resolution of $N$ is given as:

$$
\text { ... } \stackrel{y}{\longrightarrow} R \stackrel{x}{\longrightarrow} R \stackrel{y}{\longrightarrow} R \stackrel{x}{\longrightarrow} R \longrightarrow N \longrightarrow 0 .
$$

Localizing this resolution at the height-one prime ideal $\mathfrak{q}=(x, y)$ of $R$, we obtain the minimal free resolution of $N_{\mathfrak{q}}$ over $R_{\mathfrak{q}}$ :

$$
\ldots \stackrel{y}{\longrightarrow} R_{\mathfrak{q}} \stackrel{x}{\longrightarrow} R_{\mathfrak{q}} \stackrel{y}{\longrightarrow} R_{\mathfrak{q}} \stackrel{x}{\longrightarrow} R_{\mathfrak{q}} \longrightarrow N_{\mathfrak{q}} \longrightarrow 0 .
$$

This clearly shows that $\operatorname{pd}_{R_{\mathfrak{q}}}\left(N_{\mathfrak{q}}\right)=\infty$.

An $R$-module $M$ is said to be 2-Tor-rigid provided, whenever $\operatorname{Tor}_{1}^{R}(M, N)=0=\operatorname{Tor}_{2}^{R}(M, N)$ for some $R$-module $N$, we have $\operatorname{Tor}_{3}^{R}(M, N)=0$. We finish this section by noting that the conclusion of Theorem 3.1 may fail if the module $M$ is 2-Tor-rigid instead of Tor-rigid:

Example 4.7. Let $R=\mathbb{C}[\mid x, y]] /(x y), M=R /(x)$ and $N=R /\left(x^{2}\right)$. Note that each $R$-module is 2-Tor-rigid [22, 1.9]. Note also that $M \otimes_{R} N \cong M$ and hence $M \otimes_{R} N$ satisfies $\left(\widetilde{S_{v}}\right)$ for each $v \geq 0$. Also, since $R$ is reduced, $\operatorname{Tor}_{i}^{R}(M, N)$ is torsion for each $i \geq 1$. However it is easy to see that $N$ does not satisfy $\left(\widetilde{S_{1}}\right), \operatorname{Tor}_{1}^{R}(M, N) \neq 0$, and $M$ is not Tor-rigid; see [17, page 164].

It is worth noting that we do not know an example similar to Example 4.7 when $n \geq 2$. More precisely, we ask (cf. Example 4.5):

Question 4.8. Let $R$ be a hypersurface ring, and let $M$ and $N$ be nonzero $R$-modules. Assume $\operatorname{Tor}_{i}^{R}(M, N)$ is torsion for all $i \gg 0$. If $M \otimes_{R} N$ is reflexive, then must $M$ or $N$ be reflexive?

Notice, if the ring $R$ in Question 4.8 is a domain (e.g., an isolated singularity of dimension at least two), then it follows from 2.7 that both $M$ and $N$ are reflexive; see [8, 1.3].

\section{ACKNOWLEDGMENTS}

Part of this work was completed while the authors were visiting the Nesin Mathematics Village in May 2019. The authors thank the Village for providing a lively working enviroment.

The authors are grateful to W. Frank Moore for [21], and to Yongwei Yao for showing us the proof of Proposition 4.3 (ii).

\section{REFERENCES}

[1] Tokuji Araya and Yuji Yoshino, Remarks on a depth formula, a grade inequality and a conjecture of Auslander, Comm. Algebra 26 (1998), no. 11, 3793-3806. MR MR1647079 (99h:13010)

[2] Maurice Auslander and Mark Bridger, Stable module theory, Memoirs of the American Mathematical Society, No. 94, American Mathematical Society, Providence, R.I., 1969.

[3] Maurice Auslander and Idun Reiten, Applications of contravariantly finite subcategories, Adv. Math. 86 (1991), 111-152.

[4] Luchezar L. Avramov, Infinite free resolutions, six lectures on commutative algebra (Bellaterra, 1996), Progr. Math., vol. 166, Birkhäuser, Basel, 1998, pp. 1-118.

[5] Luchezar L. Avramov and Ragnar-Olaf Buchweitz, Support varieties and cohomology over complete intersections, Invent. Math. 142 (2000), no. 2, 285-318. MR MR1794064 (2001j:13017)

[6] Winfried Bruns and Jürgen Herzog, Cohen-Macaulay rings, Cambridge Studies in Advanced Mathematics, vol. 39, Cambridge University Press, Cambridge, 1993.

[7] Olgur Celikbas, Srikanth B. Iyengar, Greg Piepmeyer, and Roger Wiegand, Criteria for vanishing of tor over complete intersections, Pacific J. Math. 276 (2015), no. 1, 93-115. 
[8] Olgur Celikbas and Greg Piepmeyer, Syzygies and tensor product of modules, Math. Z. 276 (2014), no. 1-2, 457-468.

[9] Olgur Celikbas, Arash Sadeghi, and Ryo Takahashi, Bounds on depth of tensor products of modules, Journal of Pure and Applied Algebra 219 (2015), no. 5, 1670-1684.

[10] Olgur Celikbas and Ryo Takahashi, On the second rigidity theorem of Huneke and Wiegand, Proc. Amer. Math. Soc. (2019).

[11] _ Powers of the maximal ideal and vanishing of (co)homology, preprint; posted at arXiv:1901.04108v1 (2019).

[12] Mohammad T. Dibaei and Arash Sadeghi, Linkage of modules and the Serre conditions, J. Pure Appl. Algebra 219 (2015), no. 10, 4458-4478.

[13] E. Graham Evans and Phillip Griffith, Syzygies, London Mathematical Society Lecture Note Series, vol. 106, Cambridge University Press, Cambridge, 1985.

[14] Daniel R. Grayson and Michael E. Stillman, Macaulay2, a software system for research in algebraic geometry, Available at https://faculty.math.illinois.edu/Macaulay2/

[15] Melvin Hochster and Craig Huneke, Indecomposable canonical modules and connectedness, Contemporary Math. (1994), no. 159, 197-208.

[16] Craig Huneke and Roger Wiegand, Tensor products of modules and the rigidity of Tor, Math. Ann. 299 (1994), no. 3, 449-476.

[17] _ Tensor products of modules, rigidity and local cohomology, Math. Scand. 81 (1997), no. 2, 161-183.

[18] _ Correction to "Tensor products of modules and the rigidity of Tor", Math. Annalen, 299 (1994), 449-476, Mathematische Annalen 338 (2007), no. 2, 291-293.

[19] Stephen Lichtenbaum, On the vanishing of Tor in regular local rings, Illinois J. Math. 10 (1966), 220-226.

[20] Vladimir Maşek, Gorenstein dimension and torsion of modules over commutative Noetherian rings, Comm. Algebra 28 (2000), no. 12, 5783-5811, Special issue in honor of Robin Hartshorne.

[21] W. Frank Moore, Macaulay2 code to compute the biduality map and the pushforward module, unpublished (2015).

[22] M. Pavaman Murthy, Modules over regular local rings, Illinois J. Math. 7 (1963), 558-565.

Olgur Celikbas, Department of Mathematics, West Virginia University, Morgantown, WV 26506-6310, U.S.A

E-mail address: olgur.celikbas@math.wvu.edu

Hiroki Matsui, Graduate School of Mathematical Sciences, University of Tokyo, 3-8-1 Komaba, MEgURO-KU, TOKYO 153-8914, JAPAN

E-mail address: mhiroki@ms.u-tokyo.ac.jp

Arash SAdeghi, School of Mathematics, Institute for Research in Fundamental Sciences (IPM), P.O. BOX: 19395-5746, TEHRAN, IRAN.

E-mail address: sadeghiarash61@gmail.com 